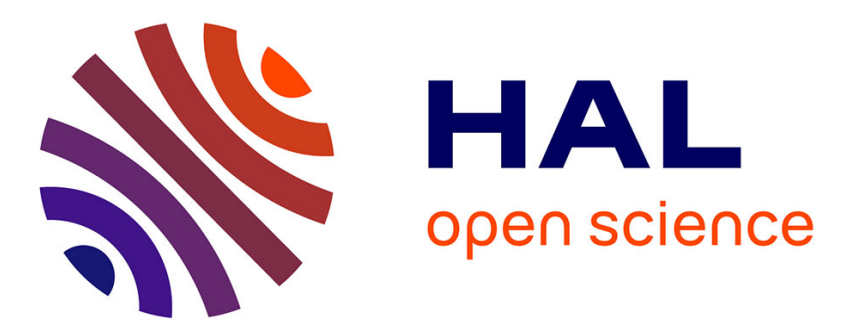

\title{
Robust Control Strategy for a Conduction-Convection System Based on the Scenario Optimization
}

\author{
Ahcène Triki, Ahmed Maidi, Karim Belharet, Jean-Pierre Corriou
}

\section{To cite this version:}

Ahcène Triki, Ahmed Maidi, Karim Belharet, Jean-Pierre Corriou. Robust Control Strategy for a Conduction-Convection System Based on the Scenario Optimization. Journal of Control, Automation and Electrical Systems, 2017, 28 (4), pp.482-492. 10.1007/s40313-017-0317-z . hal-02130566

\section{HAL Id: hal-02130566 https://hal.science/hal-02130566}

Submitted on 27 Nov 2019

HAL is a multi-disciplinary open access archive for the deposit and dissemination of scientific research documents, whether they are published or not. The documents may come from teaching and research institutions in France or abroad, or from public or private research centers.
L'archive ouverte pluridisciplinaire HAL, est destinée au dépôt et à la diffusion de documents scientifiques de niveau recherche, publiés ou non, émanant des établissements d'enseignement et de recherche français ou étrangers, des laboratoires publics ou privés. 
archives-ouvertes

\title{
Robust Control Strategy for a Conduction-Convection System Based on the Scenario Optimization
}

\author{
Jean Pierre Corriou, Ahcène Triki, Ahmed Maidi, Karim Belharet, \\ Jean-Pierre Corriou
}

\section{To cite this version:}

Jean Pierre Corriou, Ahcène Triki, Ahmed Maidi, Karim Belharet, Jean-Pierre Corriou. Robust Control Strategy for a Conduction-Convection System Based on the Scenario Optimization. Journal of Control, Automation and Electrical Systems, Springer, 2017, 28 (4), pp.482-492. 10.1007/s40313017-0317-z . hal-02130566

\section{HAL Id: hal-02130566 \\ https://hal.archives-ouvertes.fr/hal-02130566}

Submitted on 27 Nov 2019

HAL is a multi-disciplinary open access archive for the deposit and dissemination of scientific research documents, whether they are published or not. The documents may come from teaching and research institutions in France or abroad, or from public or private research centers.
L'archive ouverte pluridisciplinaire HAL, est destinée au dépôt et à la diffusion de documents scientifiques de niveau recherche, publiés ou non, émanant des établissements d'enseignement et de recherche français ou étrangers, des laboratoires publics ou privés. 


\title{
Robust control strategy for a conduction-convection system based on the scenario optimization
}

\author{
Ahcène Triki • Ahmed Maidi · Karim Belharet • Jean-Pierre \\ Corriou
}

Received: date / Accepted: date

\begin{abstract}
This paper deals with the robust control of an uncertain conduction-convection system in the framework of probabilistic control design based both on the geometric control and the scenario optimization approach. Thus, a robust control strategy that copes with parameter uncertainties is proposed for a heated rod taken as an application example of a conduction-convection system. The design approach consists in two steps. In the first step, assuming a nominal model, a state feedback that yields a stable linear lumped parameter system, of first order, in closed loop is designed by means of geometric control theory. The stability of the resulting closed loop system is demonstrated based on the perturbation theorem from semigroup theory. The second step consists in defining the input reference of the designed state feedback by a structured robust controller. The parameter tuning of the structured controller is formulated as a semi-infinite (or robust) optimization problem which is, then, relaxed using the scenario approach leading to a standard finite optimization problem. The solution of this scenario optimization problem is achieved using a genetic algorithm. The proposed control strategy is adopted to cope with parameter uncertainties in the problem of heating a steel rod. The effectiveness of the proposed robust control strategy is demonstrated by simulation.
\end{abstract}

Keywords distributed parameter system $\cdot$ geometric control $\cdot$ characteristic index $\cdot$ semigroup theory $\cdot$ robust control · semi-infinite optimization $\cdot$ scenario approach $\cdot$ PID controller

\section{Introduction}

Most physical systems are distributed in nature, i.e., the characteristic variables (states, controls and outputs) depend on several independent coordinates that are often space and time variables (Christofides, 2001; Li and Qi, 2010; Ray, 1989; Singh, 1977). These systems are termed distributed parameter systems (DPSs) and their dynamic behavior is described by partial differential equations (PDEs) involving bounded variable parameters that represent uncertainties. To ensure both stability and desired performance specifications, in closed loop, despite parameter uncertainties, a robust controller must be implemented.

Robust control theory of DPSs is an active research area and constitutes a challenging field (Christofides, 2001; Curtain and Zwart, 1995; Keulen, 1993). A survey of the different established developments in this field can be found in (Padhi and Faruque Ali, 2009). For DPSs, which are of infinite dimension, the design of a robust controller is more difficult and few contributions are reported in the literature (Armaou and Christofides, 2001; Christofides, 1998; Christofides and Baker, 1999; Christofides and Daoutidis, 1998; Ding et al., 2009). A straightforward approach, termed early lumping, consists in approximating the DPS by a lumped parameter system (LPS) commonly obtained by discretization of either the PDEs or their solution ( $\mathrm{Li}$ and Qi, 2010). The aim is to exploit the existing robust control theory for lumped parameter systems (LPSs) that provides powerful controller design methods. This approach presents the drawback that it requires an approximate LPS with a high dimensionality (order) to capture the dynamics of the original DPS. Consequently, due to the dimension of the obtained LPS, the design of the robust controller is a complex and seldom tractable task, being NP-hard (Alamo et al., 2015). An alternative approach, termed late lumping, consists in using directly the PDE model without any approximation. This approach, also, leads to NP-hard robust optimization problems, in infinite dimensional space, which remains NP-hard even though it is discretized (Borzì and Schulz, 2012).

A. Triki and A. Maidi

Laboratoire de Conception et Conduite des Systèmes de Production, Université Mouloud Mammeri, 15000 Tizi-Ouzou, Algérie E-mail: ahmed.maidi@gmail.com ;

K. Belharet

Laboratoire PRISME, Hautes Etudes d'Ingénieur campus Centre, Site Balsan, 2 allée Jean Vaillé 36000 Châteauroux;

J.-P. Corriou

Laboratoire Réactions et Génie des Procédés, UMR 7274-CNRS, Lorraine University, ENSIC. 1, rue Grandville, BP 20451, 54001 Nancy Cedex, France. 
Robust control of LPSs has attained a certain level of maturity and sophisticated design methods have been developed. For robust control of LPSs, two major approaches can be distinguished (Calafiore and Campi, 2006; Petersen and Tempo, 2014). The first approach, termed worst-case design, tries to enforce the design constraints over the domain of potential uncertainties (Calafiore and Campi, 2006; Campi et al., 2009; Toscano, 2013). This approach leads to robust (semi-infinite) optimization problems, which are classified NP-hard and their degree of complexity increases with the dimension of the system (Alamo et al., 2015; Blondel and Tsitsiklis, 1997, 2000; Toscano, 2013). The second approach, which is an interesting and promising alternative to the worst-case, is the probabilistic robust design (Alamo et al., 2015; Calafiore and Campi, 2006; Dabbene and Tempo, 2010; Tempo et al., 2013). This approach can be seen as a relaxation of the robust optimization problem by a random sampling of the constraints. In this case, a standard optimization problem with a finite number of constraints is obtained and solved with a risk of violation of the desired performances for a very small fraction of uncertainties (Calafiore and Campi, 2006; Dabbene and Tempo, 2010). Among the probabilistic robust design methods, the scenario approach is a well-established method that can tackle robust optimization problems (Calafiore and Campi, 2006; Campi et al., 2009; Tempo et al., 2013). This non-sequential approach has been applied with success to solve several control design problems (Calafiore and Campi, 2006; Campi et al., 2009).

In this work, based on the scenario approach for non-convex robust optimization problems (Grammatico et al., 2014), a robust control strategy is proposed for an uncertain conduction-convection system. To the best knowledge of the authors, the probabilistic robust design was never applied for DPSs, which makes the present work a first contribution in this field. The main idea is to overcome the NP-hardness of the robust optimization problem due to the high dimensionality of the linear diffusion-reaction equation. From this point of view, it is proposed first to design, based on the characteristic index from geometric control (Christofides and Daoutidis, 1996), a state feedback that ensures both output-tracking and stabilization in closed loop. Then, to cope with parameter uncertainties, a structured linear robust controller that defines the input reference of the state feedback is designed using the scenario approach. For the design of the structured robust controller, a non-convex robust optimization problem is formulated where the objective is the minimization of a performance index, with respect to the controller tuning parameters, subject both to stability and robustness constraints. This constrained non convex robust optimization problem is then relaxed by the scenario approach. The resulting non convex standard finite optimization problem is solved by a genetic algorithm. Note that this design approach of the structured robust controller can be applied to linear LPSs, which constitutes another contribution of the present work. The performance of the proposed control strategy is demonstrated, through simulation, in the case of problem of heating a steel rod with uncertain radius and physical parameters. The objective is to achieve a desired set point for the average temperature, along the rod, despite these parameter uncertainties.

The rest of the paper is organized as follows: the control problem of a heated rod, taken as an example of conductionconvection system is presented in Section 2. Section 3 is devoted to the proposed robust control strategy. In the first subsection, a geometric control law that enforces the output-tracking is designed in the framework of geometric control based on the characteristic index concept and the stability of the resulting closed loop system is demonstrated using the perturbation theorem from semigroup theory. In the second subsection, a design approach of a structured robust controller is proposed and applied to define the reference input of the state feedback to enhance the performance. The design problem is formulated as a semi-infinite optimization problem, which is relaxed using the scenario approach leading to a standard finite optimization problem. An application example that illustrates, by simulation, the effectiveness of the proposed control strategy, concerning the problem of heating a steel rod with uncertain radius and physical parameters, is given in Section 4 while Section 5 concludes the paper.

\section{Control problem statement}

As an example of conduction-convection system, let us consider a one dimension metal rod, of length $L$, heated with a distributed heat flux $Q^{\prime \prime}(t)$ (Figure 1). It is assumed that the rod is subject to heat exchange, across the lateral sides, with the environment and both boundaries are in contact with the surrounding medium. The energy balance, in the case of a cylindrical rod of radius $R$, yields the following model (Farlow, 1993)

$$
\begin{aligned}
\rho c_{p} \frac{\partial T(z, t)}{\partial t} & =\lambda \frac{\partial^{2} T(z, t)}{\partial z^{2}}-\frac{2 h}{R}\left(T(z, t)-T_{\mathrm{env}}\right)+d(z) Q^{\prime \prime}(t), \quad 0<z<L \\
T(0, t) & =T_{\mathrm{env}} \\
T(L, t) & =T_{\mathrm{env}} \\
T(z, 0) & =T_{0}(z)
\end{aligned}
$$

where $T(z, t) \in L^{2}(0, L)$ is the rod temperature, $z \in[0, L]$ and $t \in[0, \infty[$ are the space and time variables, respectively. $\rho, c_{p}, \lambda$ and $h$ are the rod physical properties and denote density, heat capacity, thermal conductivity and heat transfer coefficient, restrictively. $T_{\text {env }}$ is the environment temperature and $T_{0}(z) \in L^{2}(0, L)$ is the spatial temperature profile at $t=0 . Q^{\prime \prime}(t) \in L^{2}\left(\left[0, L[, \Re)\right.\right.$ is the manipulated heat flux and $d(z) \in L^{2}([0, L])$ is a known smooth function that characterizes the distribution of $Q^{\prime \prime}(t)$ on the space domain $] 0, L\left[L^{2}(0, L)\right.$ is the Hilbert space of the Lebesgue square 


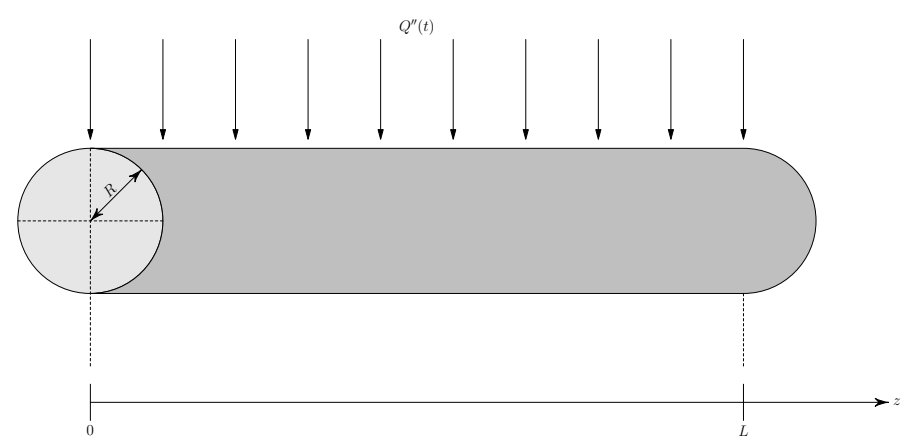

Fig. 1: Heated rod with a distributed heat flux $Q^{\prime \prime}(t)$.

integrable functions, defined on the domain space $[0, L]$, endowed with inner product (Atkinson and Han, 2009)

$$
\langle f(z), g(z)\rangle_{L^{2}(0, L)}=\int_{0}^{L} f(z) g(z) d z
$$

and the norm

Using the dimensionless variables

$$
\|f(z)\|_{L^{2}(0, L)}^{2}=\langle f(z), f(z)\rangle_{L^{2}(0, L)}
$$

$$
\theta(\xi, \tau)=\frac{T(z, t)-T_{\mathrm{env}}}{T_{f}-T_{\mathrm{env}}}, \quad \xi=\frac{z}{L}, \quad \tau=\frac{\lambda t}{\rho c_{p} L^{2}}
$$

where $T_{f}$ is the fusion temperature of the metal, the following dimensionless model results

$$
\begin{aligned}
\frac{\partial \theta(\xi, \tau)}{\partial \tau} & =\frac{\partial^{2} \theta(\xi, \tau)}{\partial \xi^{2}}-\beta \theta(\xi, \tau)+b(\xi) q^{\prime \prime}(\tau), 0<\xi<1 \\
\theta(0, \tau) & =0 \\
\theta(1, \tau) & =0 \\
\theta(\xi, 0) & =\theta_{0}(\xi)
\end{aligned}
$$

where

$$
b(\xi)=\frac{L^{2} d(\xi L)}{\lambda\left(T_{f}-T_{\mathrm{env}}\right)}, q^{\prime \prime}(\tau)=Q^{\prime \prime}\left(\frac{\rho c_{p} L^{2} \tau}{\lambda}\right), \theta_{0}(\xi)=\frac{T_{0}(z)-T_{\mathrm{env}}}{T_{f}-T_{\mathrm{env}}}
$$

and the uncertain parameter

$$
\beta=\frac{2 h L^{2}}{\lambda R}
$$

The problem addressed in this work consists in heating a rod with uncertain radius and physical parameters before crossing a rolling mill. In the following, it assumed that the heat transfer coefficient, the thermal conductivity $\lambda$ and the radius $R$ are bounded uncertain parameters within well-known regions, that is, $\left[h_{\min }, h_{\max }\right],\left[\lambda_{\min }, \lambda_{\max }\right]$ and $\left[R_{\min }\right.$, $R_{\max }$ ] leading, according to (13), to the following bounded interval for the parameter $\beta$, that is,

$$
\beta \in\left[\beta_{\min }, \beta_{\max }\right]
$$

The control objective is to design a heat flux profile $q^{\prime \prime}(\tau)$ that achieves, despite the uncertainty in the parameter $\beta$, a desired temperature for the controlled output defined as the spatial weighted average of the temperature, along the rod, expressed mathematically as follows

$$
\theta_{m}(\tau)=\int_{0}^{1} c(\xi) \theta(\xi, \tau) d \xi
$$

where $c(\xi)$ is a smooth function chosen so that the control design specifications are met. Note that the functions $b(\xi)$ and $c(\xi)$ represent key design elements for DPSs (Christofides and Daoutidis, 1996). In the present study, the choice of the two functions $b(\xi)$ and $c(\xi)$ is stated in the following Assumptions 1 and 2 .

Assumption 1 The two smooth functions $b(\xi)$ and $c(\xi)$ are chosen not orthogonal (Atkinson and Han, 2009), that is,

$$
\langle b(\xi), c(\xi)\rangle_{L^{2}(0,1)}=\int_{0}^{1} b(\xi) c(\xi) d \xi \neq 0
$$

Assumption 2 The function $c(\xi) \in H_{0}^{2}(0,1)$ with $H_{0}^{2}(0,1)$ being the Sobolev space of order 2 defined as follows (Atkinson and Han, 2009)

$$
H_{0}^{2}(0,1)=\left\{c(z) \in L^{2}(0,1): c^{(k)}(z) \in L^{2}(0,1) ; k=1,2 \text { and } c(0)=c(1)=0\right\}
$$




\section{Proposed robust control strategy}

To make the formulated robust control problem tractable and to exploit the full potential of the probabilistic control design, the idea is to use the geometric control theory. This well-developed theory, that belongs to early lumping approach (Christofides and Daoutidis, 1996; Maidi and Corriou, 2011), allows to design a state feedback that yields an uncertain LPS of first order in closed-loop. Then, to cope with parameter uncertainties of the resulting closed loop system, it is proposed to define its reference input by a structured robust controller designed using the scenario approach. These two steps are discussed at length in the following subsections.

\subsection{State feedback design}

To design the state feedback, a nominal value $\beta_{n}$ is assigned to the uncertain parameter $\beta$, which is taken equal to the mean value, that is, $\beta_{n}=\left(\beta_{\min }+\beta_{\max }\right) / 2$. The design process is based on the use of the characteristic index, from geometric control theory of DPSs, introduced by Christofides and Daoutidis (1996). Hence, the calculation of the first derivative of the controlled output (15) yields

$$
\begin{aligned}
\frac{d \theta_{m}(\tau)}{d \tau} & =\int_{0}^{1} c(\xi) \frac{\partial \theta(\xi, \tau)}{\partial \tau} d \xi \\
& =\int_{0}^{1} c(\xi)\left[\frac{\partial^{2} \theta(\xi, \tau)}{\partial \xi^{2}}-\beta_{n} \theta(\xi, \tau)+b(\xi) q^{\prime \prime}(\tau)\right] d \xi \\
& =\int_{0}^{1} c(\xi)\left[\frac{\partial^{2} \theta(\xi, \tau)}{\partial \xi^{2}}-\beta_{n} \theta(\xi, \tau)\right] d \xi+\left[\int_{0}^{1} c(\xi) b(\xi) d \xi\right] q^{\prime \prime}(\tau)
\end{aligned}
$$

Note that the manipulated heat flux $q^{\prime \prime}(\tau)$ appears linearly in the first derivative (20) of the controlled output $\theta_{m}(\tau)$. Now, since Assumption 1 holds, this means that the characteristic index of the system is $\sigma=1$. Consequently, a control law $q^{\prime \prime}(\tau)$ that preserves $\sigma=1$ between a reference input $\vartheta$ and the controlled output $\theta_{m}(\tau)$ can be obtained (Christofides and Daoutidis, 1996). In this case, the dynamics of the obtained closed loop is described by the following first order differential equation

$$
\gamma \frac{d \theta_{m}(\tau)}{d \tau}+\theta_{m}(\tau)=\vartheta
$$

where $\gamma$ is the desired time constant in closed loop and $\vartheta$ is an external input reference assumed to be constant in this work, that is, a given temperature.

By substituting $\theta_{m}(\tau)$ and $d \theta_{m}(\tau) / d \tau$ by their expressions, given by (15) and (20), respectively, into (21) and solving the resulting equation with respect to the heat flux $q^{\prime \prime}(\tau)$, the following control law results

$$
q^{\prime \prime}(\tau)=\frac{1}{\gamma \int_{0}^{1} c(\xi) b(\xi) d \xi}\left(\vartheta-\theta_{m}(\tau)-\gamma \int_{0}^{1} c(\xi)\left[\frac{\partial^{2} \theta(\xi, \tau)}{\partial \xi^{2}}-\beta_{n} \theta(\xi, \tau)\right] d \xi\right)
$$

which yields the following closed loop system

$$
\begin{aligned}
\frac{\partial \theta(\xi, \tau)}{\partial t}= & \frac{\partial^{2} \theta(\xi, \tau)}{\partial \xi^{2}}-\beta \theta(\xi, \tau)+\frac{b(\xi)}{\gamma \int_{0}^{1} c(\xi) b(\xi) d \xi} \times \\
& \left(\vartheta-\theta_{m}(\tau)-\gamma \int_{0}^{1} c(\xi)\left[\frac{\partial^{2} \theta(\xi, \tau)}{\partial \xi^{2}}-\beta_{n} \theta(\xi, \tau)\right] d \xi\right)
\end{aligned}
$$

From the closed loop dynamics (21), it follows that the external stability is ensured since the time constant $\gamma>0$. This is not sufficient, of course, to ensure the internal stability of the closed loop system (23), which must be guaranteed.

Hence, in order to derive the closed loop internal stability condition, let us first write the open-loop (8) and the state feedback (22) under the following abstract forms (Curtain and Zwart, 1995; Emirsjlow and Townley, 2000)

$$
\frac{d \theta(\tau)}{d \tau}=\mathcal{A}_{\mathrm{ol}} \theta(\tau)+\mathcal{B}_{\mathrm{ol}} q^{\prime \prime}(\tau)
$$

and

$$
q^{\prime \prime}(\tau)=-\mathcal{K} \theta(\tau)+\mathcal{H} e_{\mathrm{sf}}(\tau)
$$

where the operators are defined as follows

$$
\mathcal{A}_{\mathrm{ol}}=\frac{\partial^{2}}{\partial \xi^{2}}-\beta, \mathcal{B}_{\mathrm{ol}}=b(\xi), \mathcal{K}=I \int_{0}^{1} c(\xi)\left[\frac{\partial^{2}}{\partial \xi^{2}}-\beta_{n}\right] d \xi, \mathcal{H}=I \gamma^{-1}
$$

with $I=\left[\int_{0}^{1} c(\xi) b(\xi) d \xi\right]^{-1}$ and $e_{\mathrm{sf}}(\tau)=\vartheta-\theta_{m}(\tau)($ sf $=$ state feedback $)$ 
Hence, since $\vartheta$ is a constant temperature, the closed loop system (23) can be rewritten as an interconnection, in abstract form, as follows

$$
\begin{aligned}
\frac{d e_{\mathrm{sf}}(\tau)}{d \tau} & =-\frac{1}{\gamma} e_{\mathrm{sf}}(\tau) \\
\frac{d \theta(\tau)}{d \tau} & =\mathcal{A}_{\mathrm{cl}} \theta(\tau)+\mathcal{H} e_{\mathrm{sf}}(\tau)
\end{aligned}
$$

where $\mathcal{A}_{\mathrm{cl}}=\left(\mathcal{A}_{\mathrm{ol}}-\mathcal{B}_{\mathrm{ol}} \mathcal{K}\right)$ is the resulting state operator in closed loop.

From (21), since $\gamma>0$ (time constant), it follows that

$$
\left\|e_{\mathrm{sf}}(\tau)\right\| \leq\left|e_{\mathrm{sf}}(0)\right| e^{-\tau / \gamma}
$$

consequently, the closed loop system (27)-(28) is internally stable if the state operator $\mathcal{A}_{\text {cl }}$ generates a stable semigroup (Christofides and Daoutidis, 1996). The internal stability condition of the closed loop system (23) is provided by Proposition 1.

Proposition 1 The closed loop system (27)-(28) for which Assumptions 1 and 2 hold is internally stable, that is, the operator $\mathcal{A}_{c l}$ generates an exponentially stable semigroup if

$$
|I|\left\|\frac{d^{2} c(\xi)}{d \xi^{2}}-\beta_{n}\right\|_{L^{2}(0,1)}\left\|b^{2}(\xi)\right\|_{L^{2}(0,1)}<\beta_{\min }+\pi^{2}
$$

Proof The resulting closed loop state operator $\mathcal{A}_{\mathrm{cl}}$ represents the open-loop state operator $\mathcal{A}_{\mathrm{ol}}$ perturbed by the operator $\mathcal{B}_{o l} \mathcal{K}$. Thus, according to the bounded operator theorem (Engel and Nagel, 2006, Page 116), since $\mathcal{A}_{\mathrm{ol}}$ is a generator of a stable semigroup (Afifi et al., 2012), that is,

$$
\|U(\tau)\| \leq e^{-\left(\beta+\pi^{2}\right) \tau}, \quad(\beta>0 \text { according to }(13))
$$

and if the perturbation $\mathcal{B}_{\mathrm{ol}} \mathcal{K}$ is bounded, then the operator $\mathcal{A}_{\mathrm{cl}}$ is a generator of the following semigroup

$$
\|S(\tau)\| \leq e^{\left(-\beta-\pi^{2}+\left\|\mathcal{B}_{\mathrm{ol}} \mathcal{K}\right\|_{L^{2}(0,1)}\right) \tau}
$$

which is stable if the following condition

$$
\left.-\left(\beta+\pi^{2}\right)+\| \mathcal{B}_{\mathrm{ol}} \mathcal{K}\right) \|_{L^{2}(0,1)}<0
$$

holds.

The linear operator $\mathcal{B}_{\mathrm{ol}} \mathcal{K}$ is bounded, that amounts to verify the existence of the constant $C$ such that

$$
\left\|\mathcal{B}_{\mathrm{ol}} \mathcal{K} \theta(\tau)\right\|_{L^{2}(0,1)} \leq C\|\theta(\tau)\|_{L^{2}(0,1)}
$$

and the smallest $C$ is the norm of $\left\|\mathcal{B}_{\mathrm{ol}} \mathcal{K}\right\|_{L^{2}(0,1)}$.

The first step of the proof is to demonstrate that the operator $\mathcal{B}_{\mathrm{ol}} \mathcal{K}$ is bounded. Calculating the norm of $\mathcal{B}_{\mathrm{ol}} \mathcal{K}$ gives

$$
\begin{aligned}
\left\|\mathcal{B}_{\mathrm{ol}} \mathcal{K} \theta(\tau)\right\|_{L^{2}(0,1)}^{2} & =\left\|I b(\xi) \int_{0}^{1} c(\xi)\left[\frac{\partial^{2} \theta(\xi, \tau)}{\partial \xi^{2}}-\beta_{n} \theta(\xi, \tau)\right] d \xi\right\|_{L^{2}(0,1)}^{2} \\
& =\int_{0}^{1}\left[I b(\xi) \int_{0}^{1} c(\xi)\left[\frac{\partial^{2} \theta(\xi, \tau)}{\partial \xi^{2}}-\beta_{n} \theta(\xi, \tau)\right] d \xi\right]^{2} d \xi \\
& =I^{2} \int_{0}^{1}\left[b(\xi) \mid \int_{0}^{1} c(\xi)\left[\frac{\partial^{2} \theta(\xi, \tau)}{\partial \xi^{2}}-\beta_{n} \theta(\xi, \tau)\right] d \xi\right]^{2} d \xi
\end{aligned}
$$

By taking into account assumption (2) and boundary conditions (9) and (10), the integration by parts of the first term of the internal integral in (37) yields

$$
\left\|\mathcal{B}_{\mathrm{ol}} \mathcal{K} \theta(\tau)\right\|_{L^{2}(0,1)}^{2}=I^{2} \int_{0}^{1}\left[b(\xi)\left|\int_{0}^{1}\left(\frac{d^{2} c(\xi)}{d \xi^{2}}-\beta_{n}\right) \theta(\xi, \tau) d \xi\right|\right]^{2} d \xi
$$

Now, Cauchy-Schwartz inequality allows to write

$$
\left|\int_{0}^{1}\left(\frac{d^{2} c(\xi)}{d \xi^{2}}-\beta_{n}\right) \theta(\xi, \tau) d \xi\right| \leq \int_{0}^{1}\left\|\frac{d^{2} c(\xi)}{d \xi^{2}}-\beta_{n}\right\|_{L^{2}(0,1)}\|\theta(\xi, \tau)\|_{L^{2}(0,1)} d \xi
$$




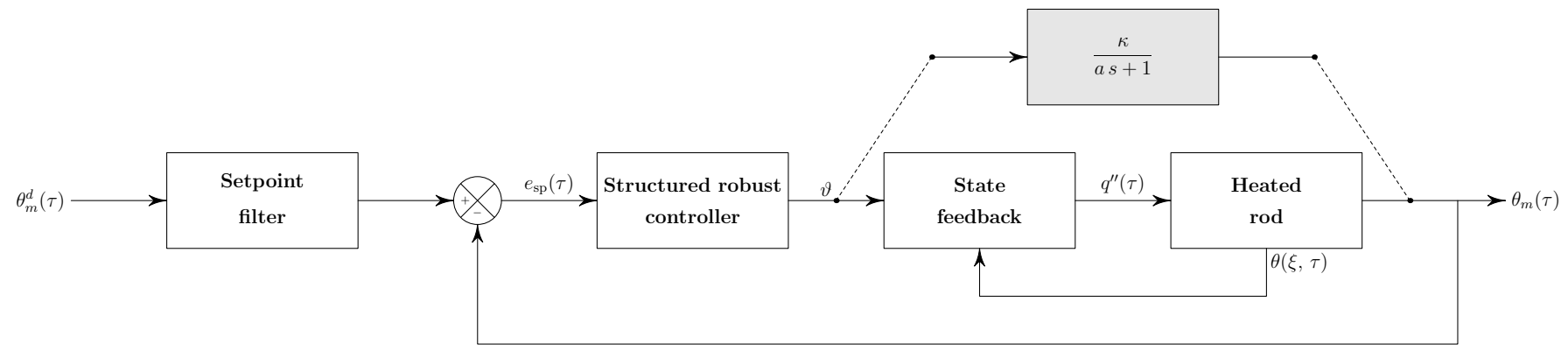

Fig. 2: Proposed robust control strategy (dotted lines mean equivalence).

hence

$$
\begin{aligned}
\left\|\mathcal{B}_{\mathrm{ol}} \mathcal{K} \theta(\tau)\right\|_{L^{2}(0,1)}^{2} & \leq I^{2} \int_{0}^{1} b^{2}(\xi)\left\|\frac{d^{2} c(\xi)}{d \xi^{2}}-\beta_{n}\right\|_{L^{2}(0,1)}^{2}\|\theta(\xi, \tau)\|_{L^{2}(0,1)}^{2} d \xi \\
\left\|\mathcal{B}_{\mathrm{ol}} \mathcal{K} \theta(\tau)\right\|_{L^{2}(0,1)} & \leq|I|\left\|b^{2}(\xi)\right\|_{L^{2}(0,1)}\left\|\frac{d^{2} c(\xi)}{d \xi^{2}}-\beta_{n}\right\|_{L^{2}(0,1)}\|\theta(\xi, \tau)\|_{L^{2}(0,1)} \\
& \leq C\|\theta(\xi, \tau)\|_{L^{2}(0,1)}
\end{aligned}
$$

where

$$
C=|I|\left\|\frac{d^{2} c(\xi)}{d \xi^{2}}-\beta_{n}\right\|_{L^{2}(0,1)}\left\|b^{2}(\xi)\right\|_{L^{2}(0,1)}
$$

Consequently, it can be concluded that the operator $\mathcal{B}_{\mathrm{ol}} \mathcal{K}$ is bounded and its norm is $\left\|\mathcal{B}_{\mathrm{ol}} \mathcal{K}\right\|_{L^{2}(0,1)}=C$.

The second step of the proof is to derive the internal stability condition (30). Consequently, according to the above development, the closed loop state operator $\mathcal{A}_{\mathrm{cl}}$ generates the semigroup

$$
\|S(\tau)\| \leq e^{-\left(\beta+\pi^{2}-C\right) \tau}
$$

which is stable if $\beta+\pi^{2}-C>0$, that is,

$$
|I|\left\|\frac{d^{2} c(\xi)}{d \xi^{2}}-\beta_{n}\right\|_{L^{2}(0,1)}\left\|b^{2}(\xi)\right\|_{L^{2}(0,1)}<\beta+\pi^{2}
$$

Since $0<\beta_{\min } \leq \beta \leq \beta_{\max }$, it follows that the closed loop system (27)-(28) is exponentially stable if

$$
|I|\left\|\frac{d^{2} c(\xi)}{d \xi^{2}}-\beta_{n}\right\|_{L^{2}(0,1)}\left\|b^{2}(\xi)\right\|_{L^{2}(0,1)}<\beta_{\min }+\pi^{2}
$$

Remark 1 The left-hand side of condition (46) depends on both $b(\xi)$ and $c(\xi)$, hence it can be checked by an appropriate choice of these two functions which represent key elements for the control design.

Now, the designed control law (22) achieves an exact output-tracking with the desired dynamics characterized by the time constant $\gamma$ when $\beta=\beta_{n}$. In the case of deviation of $\beta$ from the nominal value $\beta_{n}$, the closed loop dynamics characterized by (21) will not be exactly achieved and a deterioration of the performance will be observed on the controlled output $\theta_{m}(\tau)$. Thus, the settling time will be affected and important bounded steady-state errors will occur (this is illustrated in the case of the example studied in Section 4). Consequently, the closed loop system can be described by the following uncertain continuous transfer function

$$
G(s, \delta)=\frac{\Theta_{m}(s)}{\mathcal{V}(s)}=\frac{\kappa}{a s+1}
$$

with $a \in\left[a_{\min }, a_{\max }\right]$ and $k \in\left[\kappa_{\min }, \kappa_{\max }\right]$ denote the bounding intervals for the uncertainties $a$ and $\kappa$, respectively. These uncertainties are grouped in the vector $\delta \in \Delta$, that is, $\delta=[a, \kappa] . \Theta_{m}(s)$ and $\mathcal{V}(s)$ are the Laplace transforms of the controlled output $\theta_{m}(\tau)$ and the reference input $\vartheta(\tau)$, respectively.

Since the upper and lower bounds of the uncertain parameters $a$ and $\kappa$ cannot be determined analytically as functions of $\beta$, one can estimate them from the observed output $\theta_{m}(\tau)$ by assuming several values of the deviation of the parameter $\beta$. This can be easily done through simulation.

To avoid the deterioration of the performance in spite of the variation of the parameters $a$ and $\kappa$, it is proposed to define the reference input $\vartheta$, of the state feedback (22), by an external structured robust controller as shown by Figure 2 that summarizes the adopted control strategy. To design the robust controller, it is proposed to use the scenario optimization following a proposed approach developed in Subsection 3.2. 


\subsection{External robust controller design}

In this subsection, a design approach of a structured robust controller for a LPS is developed. The approach consists, first, in formulating the design specifications as a semi-infinite (or robust) optimization problem, which will be then solved using the scenario approach (Calafiore and Campi, 2006; Campi et al., 2009).

\subsubsection{Formulation of the controller design as a semi-infinite optimization problem}

To design the structure robust controller $G_{c}(s, p)$, that is, to determine the tuning parameters $p\left(p \in \Re^{n_{p}}\right)$, it is proposed to minimize a given performance index. In the following development, the Integral of Square Error (ISE) is assumed. The ISE is defined as follows

$$
J(p, \delta)=\int_{0}^{\infty} e_{\mathrm{sp}}^{2}(\tau) d \tau
$$

where the tracking error is $e_{\mathrm{sp}}(\tau)=\theta_{m}^{d}(\tau)-\theta_{m}(\tau)$ (sp = set point) with $\theta_{m}^{d}(\tau)$ the desired set point of the controlled output $\theta_{m}(\tau)$. Note that the value of the ISE depends both on the controller parameters $p$ and the system uncertainties $\delta$.

Assumption 3 In the following development, it is assumed that the controller $G_{c}(s, p)$ includes an integral action, that is, the tracking error $e_{s p}(\tau)$ for a setup input $\theta_{m}^{d}(\tau)$ is zero, which implies that the value of the ISE (48) is finite.

Our objective is to seek controller parameters $p$ that ensure best performance by minimizing the ISE whatever the system uncertainties $\delta$. Thus, the design of the robust controller $G_{c}(s, p)$ can be formulated as a min-max optimization problem as follows

$$
\min _{p} \max _{\delta} J(p, \delta)
$$

where $J(p, \delta)$ is the objective function.

In addition, the controller parameters $p$ can be constrained by assuming robustness and stability constraints of inequality kind. In this case, the controller design optimization problem takes the following form

$$
\min _{p} \max _{\delta} J(p, \delta)
$$

subject to:

$$
\begin{aligned}
& g^{r}(p, \delta) \leq 0 \\
& g^{s}(p, \delta) \leq 0
\end{aligned}
$$

where $g^{r}$ and $g^{s}$ are the robustness and stability constraints, respectively. These constraint functions are the relations between the controller parameters $p$ and the uncertain parameters $\delta$ defined so as to meet both robustness and stability specifications. For instance to define the stability constraints $g^{s}(p, \delta)$, the Routh-Jury criterion (Corriou, 2004) can be used. 2009)

The formulated min-max optimization problem (50)-(52) can be rewritten under the following form (Campi et al.,

$$
\min _{p, w} w
$$

subject to:

$$
\begin{aligned}
& J(p, \delta) \leq w \\
& g^{r}(p, \delta) \leq 0 \\
& g^{s}(p, \delta) \leq 0
\end{aligned}
$$

which is a semi-infinite optimization problem (finite number of optimization variables $p$ and infinite number of constraints due the uncertainties $\delta$ ) termed also robust optimization problem, which is NP-hard (Blondel and Tsitsiklis, 1997, 2000) and generally non-convex.

Let us first determine the analytical expression of the performance index $J(p, \delta)$. The transfer function between the step input $\theta_{m}^{d}(\tau)$ and the tracking error $e_{\mathrm{sp}}(\tau)$ is given as follows

$$
\frac{E_{\mathrm{sp}}(s)}{\Theta_{m}^{d}(s)}=\frac{1}{1+G_{c}(s, p) G(s, \delta)}
$$

where $E_{\mathrm{sp}}(s)$ and $\Theta_{m}^{d}(s)$ are the Laplace transforms of tracking error $e_{\mathrm{sp}}(\tau)$ and the external input reference $\theta_{m}^{d}(\tau)$, respectively.

The value of the ISE (48) can be evaluated using the Laplace transform properties as follows (Åström, 1970; Puri and Weygandt, 1964)

$$
J(p, \delta)=\frac{1}{2 \pi j} \int_{-j \infty}^{+j \infty} E_{\mathrm{sp}}(s) E_{\mathrm{sp}}(-s) d s
$$


To evaluate this complex integral, in general, there are two methods: the transfer function and the state-space methods. These methods are discussed by Puri and Weygandt (1964), Åström (1970) and Borne and Rotella (1996).

Once the analytical expression of the objective function $J(p, \delta)$ is determined, the robust optimization problem (53)-(56) can be transformed into a standard finite optimization problem, with a finite number of constraints, using the scenario approach based on the sampling of constraints (Calafiore and Campi, 2006; Campi et al., 2009).

\subsubsection{Solving the semi-indefinite optimization problem using the scenario approach}

The Scenario approach is a probabilistic tool used to solve semi-infinite optimization problems. Its principle consists in deriving a standard optimization problem with a finite number of constraints by assuming a random sampling process of the constraints. In this approach, a probability measure of the uncertainty is assumed over the uncertainty domain and the sample set is generated according to this measure (Calafiore and Campi, 2006; Campi et al., 2009).

By using the scenario approach, the solution of the formulated non-convex robust optimization problem (53)-(56) is reduced to the solution of the following standard optimization problem (Alamo et al., 2015; Campi et al., 2009; Toscano, 2013)

$$
\min _{p, w} w
$$

subject to:

$$
\begin{aligned}
& J\left(p, \delta^{(i)}\right) \leq w \\
& g^{r}\left(p, \delta^{(i)}\right) \leq 0 \\
& g^{s}\left(p, \delta^{(i)}\right) \leq 0, \quad i=1, \ldots, N
\end{aligned}
$$

where $\delta^{(i)}=\left[a^{(i)}, \kappa^{(i)}\right]$ are the independent identically distributed samples, generated according to the assumed probability measure, and $N$ is the number of samples.

In this case, the solution $p^{*}$ of the scenario optimization (59)-(62) satisfies all constraints with a probability not smaller than $1-\eta, \eta \in(0,1)$, with an expectation of constraint violation probability not larger than $\varepsilon(\varepsilon \in(0,1))$. $\varepsilon$ and $\eta$ are termed violation and confidence parameters, respectively, that constitute the design parameters of the scenario approach that fix the number of samples $N$ (Calafiore and Campi, 2006; Campi et al., 2009).

The number of samples $N$ plays a key role in solving robust optimization problems with a desired probabilistic specification $(\eta$ and $\varepsilon$ ). For convex optimization problems, several bounds for the sample size are proposed and refined in the literature (Alamo et al., 2015; Calafiore and Campi, 2006; Campi et al., 2009). On the other hand, the sample size of a non convex optimization problems remains very challenging. This issue has not been widely investigated and only few interesting results are reported in the literature (Alamo et al., 2009; Grammatico et al., 2014). In this work, the bound on the sample size $N$ developed recently by Grammatico et al. (2014) is used to relax the non convex robust optimization problem (59)-(62). This bound is given by the following formula (Grammatico et al., 2014, Corollary 1)

$$
N \geq \frac{\frac{\exp (1)}{\exp (1)-1}(d+1)}{\varepsilon}\left(d-1+\ln \left(\frac{M}{\eta}\right)\right)
$$

where the integer $M$ satisfies $M \geq d+1$.

It is worth noticing that the bound (63) is easy to determine since it is a function of the parameters $\varepsilon, \eta$ and the number of the optimization variables $d$, of the scenario optimization problem (59)-(62), that is, $d=n_{p}+1$ (the number of the tuning parameters $n_{p}$ of the structured robust controller $G_{c}(s, p)$ plus the introduced optimization variable $\left.w\right)$.

\section{Application example}

In this section, the tracking performance of the proposed robust control strategy is demonstrated through numerical simulation runs. The rod is made of steel that has the physical properties (Taler and Duda, 2006$): \rho=7350.8 \mathrm{~kg} \cdot \mathrm{m}^{-3}$, $c_{p}=570 \mathrm{~J} \cdot \mathrm{kg}^{-1} \cdot \mathrm{K}^{-1}$ and $h=4.19 \mathrm{~W} \cdot \mathrm{m}^{-2} \cdot \mathrm{K}^{-1}$. The rod length is $L=1 \mathrm{~m}$ while the radius $R$ is uncertain with the bounded interval $[0.15 \mathrm{~m}, 0.35 \mathrm{~m}]$, which gives, according to $(13), \beta \in[0.6667,1.3317]$. The distribution function of the heat flux is $b(\xi)=1$ (uniform distribution) and the sensing function $c(\xi)=\xi(1-\xi)$. The assumed data leads, according to $(22)$, to the following state feedback

$$
q^{\prime \prime}(\tau)=\frac{6}{\gamma}\left(\vartheta-\theta_{m}(\tau)-\gamma \int_{0}^{1} \xi(1-\xi)\left[\frac{\partial^{2} \theta(\xi, \tau)}{\partial \xi^{2}}-\beta_{n} \theta(\xi, \tau)\right] d \xi\right)
$$

The closed loop system is simulated using the method of lines (Van de Wouwer et al., 2001) by assuming $a=3$ and $\beta_{n}=0.8889$ (the nominal value $\beta_{n}$ is obtained using (13) for $R=R_{n}$ with the nominal radius $R_{n}=\left(R_{\min }+R_{\max }\right) / 2$ ).

The first simulation run, consists in determining the bounded intervals for the uncertain parameters $a$ and $\kappa$. Thus, the input reference $\vartheta$ is taken equal to 0.5 and several values of $\beta$, linearly spaced between its bounds, are 


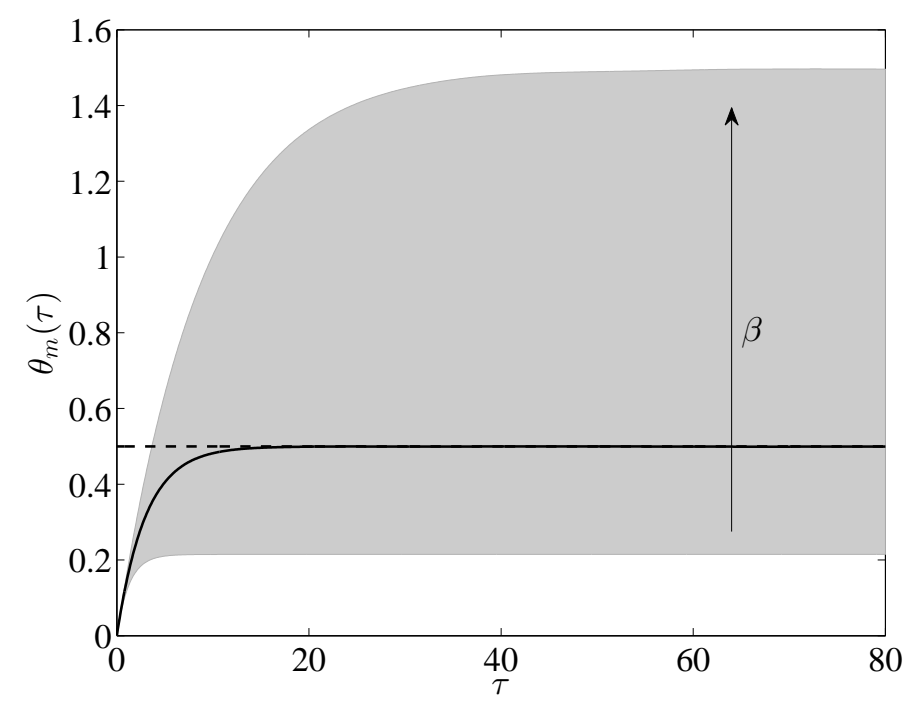

Fig. 3: Performance of the state feedback control law (64). The solid line is the controlled output $\theta_{m}(\tau)$, dotted line is the input reference $\vartheta$ and the filled area (in gray) is the variation domain of the controlled output $\theta_{m}(\tau)$ for all possible uncertainties in $\delta \in \Delta$.

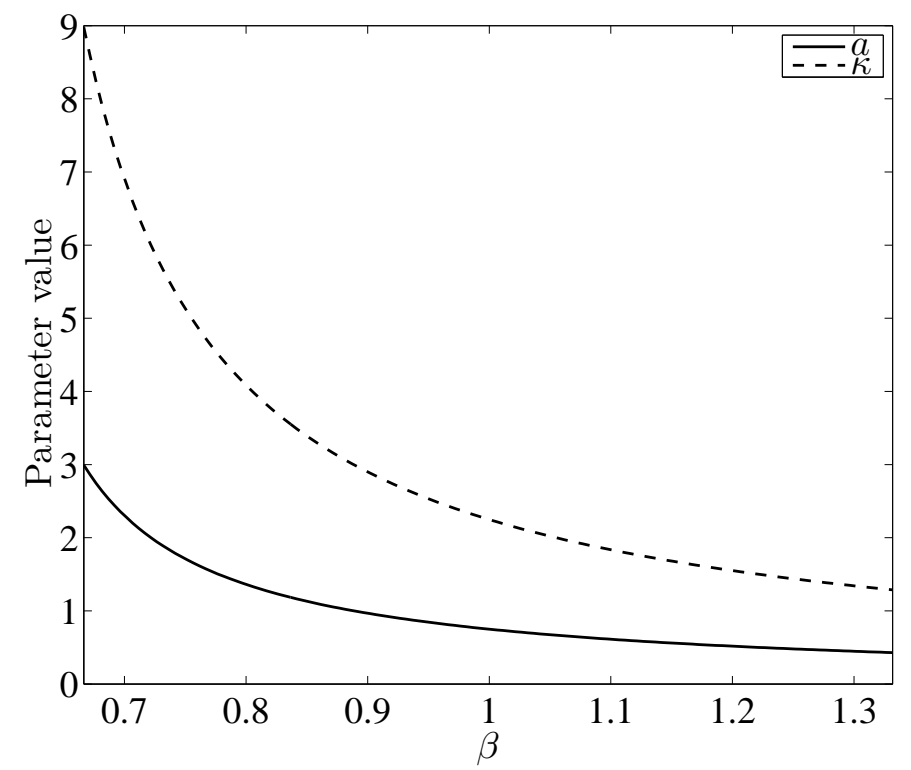

Fig. 4: Variations of the model parameters $a$ and $\kappa$ with respect to the uncertain parameter $\beta$.

considered. The evolution of the controlled output $\theta_{m}(\tau)$ is given by Figure 3 . The obtained results show clearly that in the presence of uncertainties $\beta \neq \beta_{n}$, the designed state feedback (64) is unable to achieve the set point tracking, that is, the controlled output $\theta_{m}(t)$ does not track its set point $\vartheta$. Note that the set point tracking is achieved only when $\beta=\beta_{n}$, which is expected because the state feedback (64) is designed by considering the nominal value $\beta_{n}$ of the uncertain parameter $\beta$. The evolutions of the parameters $a$ and $\kappa$, of the uncertain model (47), with respect the variation of the parameter $\beta$ are depicted in Figure 4, which shows a nonlinear evolution of both parameters. The bounded intervals are identified as $a \in[0.4293,2.9925]$ and $\kappa \in[1.2872,8.9770]$ leading to $\delta=[a, \kappa] \in \Delta=[0.4293,2.9925] \times[1.2872,8.9770] \in$ $\Re^{2}$.

Now, let us define the input reference $\vartheta$ by means of a structured controller. The PID controller remains the widely used structured controller in industry (Toscano, 2013). In this section, the proposed design approach, based on the scenario optimization, is applied to design this kind of structured controller for the resulting LPS (21).

The transfer function of the PID controller is

$$
G_{c}(s, p)=K_{c}\left(1+\frac{1}{T_{i} s}+T_{d} s\right)
$$

where $K_{c}, T_{i}$ and $T_{d}$ denote the tuning parameters, that is, the gain, integral constant and derivative constant, respectively. Thus, $p=\left[K_{c}, T_{i}, T_{d}\right]$. 
The Laplace transform of the tracking error is

$$
E_{\mathrm{sp}}(s)=\frac{T_{i} \kappa s+T_{i}}{T_{i}\left(\kappa+a K_{c} T_{d}\right) s^{2}+T_{i}\left(1+a K_{c}\right) s+a K_{c}}
$$

The evaluation of ISE (48), using the Åström method (Åström, 1970), yields

$$
J\left(K_{c}, T_{i}, T_{d}, \delta\right)=\frac{T_{i}}{2\left(1+a K_{c}\right)}\left(\frac{1}{a K_{c}}+\frac{\kappa^{2}}{T_{i}\left(\kappa+a K_{c} T_{d}\right)}\right)
$$

with $\delta \in \Delta$.

In addition to the choice of the controller structure, some robustness constraints on its tuning parameters $p$ can be imposed (Aström and Hädgglund, 2006). These constraints define the robustness region. For instance, for a commercial PID controller, the time constants are constrained by (Åström and Hädgglund, 2006)

$$
g^{r}\left(K_{c}, T_{i}, T_{d}, \delta\right): T_{d}-\frac{T_{i}}{4} \leq 0
$$

To ensure the stability of the robust control strategy, the stability constraints are derived based on the Routh-Jury criterion (Corriou, 2004), which yields the following constraints

$$
g^{r}\left(K_{c}, T_{i}, T_{d}, \delta\right)=-K_{c} \leq 0
$$

In addition, the two time constants $T_{i}$ and $T_{d}$ must be positive, that is,

$$
T_{i}>0, \quad T_{d}>0
$$

The scenario optimization problem to solve, to determine the robust PID controller parameters, is summarized as follows

$$
\min _{K_{c}, T_{i}, T_{d}, w} w
$$

subject to:

$$
\begin{aligned}
\left.\frac{T_{i}}{2\left(1+a^{(i)} K_{c}\right)} \quad \frac{1}{a^{(i)} K_{c}}+\frac{\left(\kappa^{(i)}\right)^{2}}{T_{i}\left(\kappa^{(i)}+a^{(i)} K_{c} T_{d}\right)}\right) & \leq w, \quad i=1, \ldots, N \\
T_{d}-\frac{T_{i}}{4} & \leq 0 \\
-K_{c} & \leq 0 \\
-T_{i} & \leq 0 \\
-T_{d} & \leq 0
\end{aligned}
$$

where $N=12093$ is the number of samples, obtained using the formula (63), that corresponds to $\varepsilon=10^{-2}$ and $\eta=10^{-3}$. The samples are generated by assuming a uniform probability measure. Note that the number of decision variables of $(71)$ is $d=4\left(K_{c}, T_{i}, T_{d}\right.$ and $\left.w\right)$ and $M$ is taken equal to $d+1$, i.e., $M=5$.

The scenario optimization problem (71) is solved using the genetic algorithm, which yields the following solution:

$$
K_{c}^{*}=24.2867, T_{i}^{*}=13.0724, T_{d}^{*}=3.1732 \text { and } w^{*}=0.1353
$$

Figure 5 gives the different values of the performance index (67) for all possible uncertainties of the parameters $a$ and $\kappa$. It follows that, with the obtained optimal tuning (72), the first constraint of (71) is violated for a very small portion of $\Delta$, that is, when $a$ and $\kappa$ are very close to 0.4293 and 8.9770 , respectively. In this case, since the constraint is not significantly violated, a low performance deterioration is observed in the output response. Note that, in the simulation run, the desired set point $\theta_{m}^{d}(\tau)$ is filtered by a first-order filter to avoid sudden fluctuations of the controlled output $\theta_{m}(\tau)$ (see Figure 2). The evolution of both output $\theta_{m}(\tau)$ and manipulated heat flux $q^{\prime \prime}(\tau)$ in closed loop for a set of uncertainties generated randomly are given by Figure 6 . The reported results show and demonstrate the robustness of the proposed control strategy. It is clear that despite the parameter uncertainty, the output-tracking is achieved with low and tolerable deviations with respect to the nominal response. The moves of the manipulated heat flux $q^{\prime \prime}(\tau)$ remain also physically reasonable whatever the deviation of the parameter $\beta$.

Remark 2 In this application, it is assumed that $R \in[0.15,0.35]$ leading to $\beta \in[0,6667,1.3317]$, our aim is to give an example, which is correct from a practical point of view. Therefore reasonable physical limit values of $R$ are assumed. It is worth noting that the proposed strategy performs well even for large uncertainty of $\beta$. 


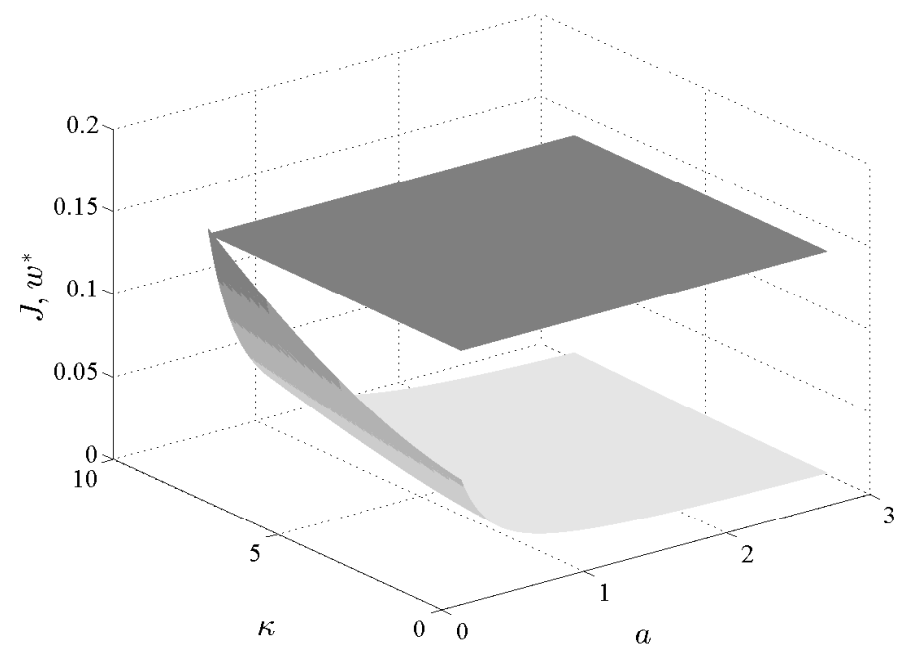

Fig. 5: Values of the of performance index (67) for random uncertainties. The flat surface represents $w^{*}$ whereas the second surface is $J$.
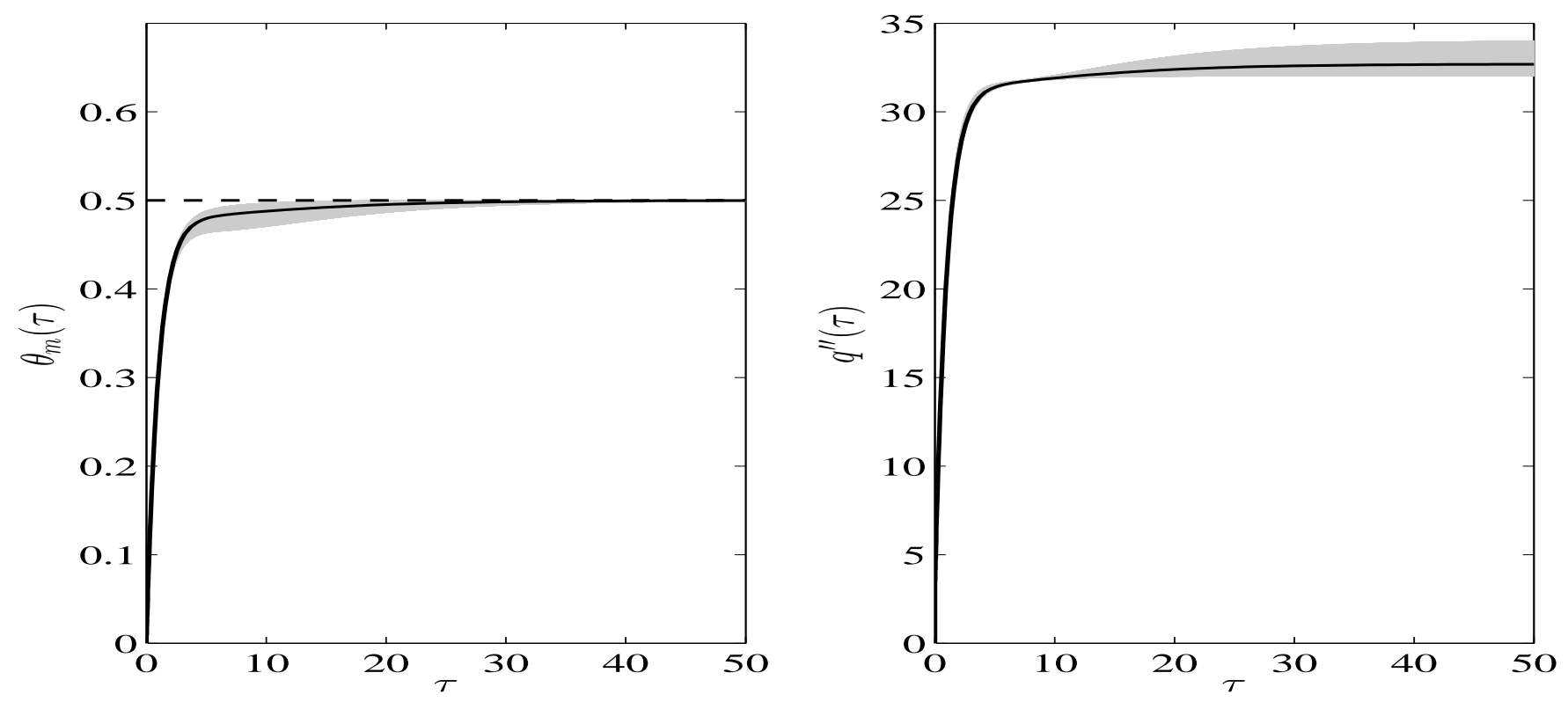

Fig. 6: Performance of the proposed robust control strategy. Left : solid line is the nominal response $\left(\beta=\beta_{n}\right)$ of the controlled output $\theta_{m}(\tau)$ and its variation domain (filled area in gray) in the case of uncertainties $\left(\beta \neq \beta_{n}\right)$. Right : evolution of the manipulated heat flux $q^{\prime \prime}(\tau)$. Solid line for $\beta=\beta_{n}$ (nominal case) and filled area in gray for $\beta \neq \beta_{n}$.

\section{Conclusion}

In this paper, a robust control strategy for a heated rod, modeled by a linear diffusion-reaction equation, with bounded parameter uncertainties is developed by means of late lumping approach. First, the system is lumped by means of a state feedback designed in the framework of geometric control using the characteristic index concept. It is demonstrated that by an appropriate choice of the sensing and actuation functions, the resulting closed loop system is exponentially stable despite the bounded parameter uncertainties. Nevertheless, the output-tracking is significantly affected when the system parameters deviate from their nominal values. Thus, to achieve a perfect output-tracking, in the presence of uncertainties, it is proposed to define the input reference of the state feedback by a structured robust controller. From this point of view, a designed approach of a robust structured controller for LPS is proposed. By assuming that the structure of the robust controller is well known and includes an integral action, the parameter tuning problem of the structured controller is formulated as a semi-infinite optimization that guarantees both robustness and stability in closed loop. Then, the formulated robust optimization problem is relaxed using the scenario approach. The performance of the developed control strategy is demonstrated by an application example that concerns the heating problem of a steel rod with uncertain radius and physical parameters. The simulation results show the effectiveness of the developed control strategy in coping with parameter uncertainties. 
The probabilistic control design approach is a promising framework for robust control. This approach fully relaxes the design problem and allows to overcome the complexity and the intractability of the robust control problems. To the best of our knowledge, the present work is the first attempt to apply the probabilistic control design approach for a DPS. The results obtained, in our opinion, are conclusive and open the opportunity to investigate this field in the case of DPS with parameters that exhibit spatio-temporal variation, which is under investigation of the authors.

\section{References}

L. Afifi, A. El Jai, and E. Zerrik. Systems Theory Regional Analysis of Infinite-Dimensional Linear Systems. Presses Universitaires de Perpignan, 2012.

T. Alamo, R. Tempo, and E. F. Camacho. Randomized strategies for probabilistic solutions of uncertain feasibility and optimization problems. IEEE Transactions on Automatic Control, 54(11):2545-2559, 2009.

T. Alamo, R. Tempo, A. Luque, and D. R. Ramirez. Randomized methods for design of uncertain systems: Sample complexity and sequential algorithms. Automatica, 52(2):160-172, 2015.

A. Armaou and P. D. Christofides. Robust control of parabolic pde systems with time-dependent spatial domains. Automatica, 37(1):61-69, 2001.

K. J. Åström. Introduction to Stochastic Control Theory. Academic Press, New York, 1970.

K. J. Åström and T. Hädgglund. Advanced PID Control. Instrumentation, Systems, and Automation Society (ISA), 2006.

K. Atkinson and W. Han. Theoretical numerical Analysis. A functional analysis framework, volume 39 of Texts in Applied Mathematics Series. Springer-Verlag, third edition, 2009.

V. Blondel and J. N. Tsitsiklis. NP-hardness of some linear control design problems. SIAM Journal on Control and Optimization, 35(6):2118-2127, 1997.

V. D. Blondel and J. N. Tsitsiklis. A survey of computational complexity results in systems and control. Automatica, 36(9):1249-1274, 2000.

P. Borne and F. Rotella. Commande optimale. In Traité Mesures et Contrôle, $R$ 7 427, pages 1-12. Techniques de l'Ingénieur, 1996.

A. Borzì and V. Schulz. Computational Optimization of Systems Governed by Partial Differential Equations. SIAM, Philadelphia, USA, 2012.

G. C. Calafiore and M. C. Campi. The scenario approach to robust control design. IEEE Transactions on Automatic Control, 51(5):742-753, 2006.

M. C. Campi, S. Garatti, and M. Prandini. The scenario approach for systems and control design. Annual Reviews in Control, 33(2):149-157, 2009.

P. D. Christofides. Robust control of parabolic PDE systems. Chemical Engineering Science, 53(16):2949-2965, 1998.

P. D. Christofides. Nonlinear and robust control of PDE systems: methods and applications to transport-reaction processes. Birkhaüser, Boston, 2001.

P. D. Christofides and J. Baker. Robust output feedback control of quasi-linear parabolic pde systems. Systems and Control Letters, 36(5):307-316, 1999.

P. D. Christofides and P. Daoutidis. Feedback control of hyperbolic PDE systems. AIChE Journal, 42(11):3063-3086, 1996.

P. D. Christofides and P. Daoutidis. Robust control of hyperbolic PDE systems. Chemical Engineering Science, 53 (1):85-105, 1998.

J. P. Corriou. Process control - Theory and applications. Springer, London, 2004.

R. F. Curtain and H. Zwart. An Introduction to Infinite-Dimensional Linear Systems Theory. Springer, New York, 1995.

F. Dabbene and R. Tempo. Probabilistic and randomized tools for control design. In W. S. Levine, editor, The Control Handbook. Taylor and Francis, London, second edition, 2010.

L. Ding, A. Johansson, and T. Gustafsson. Application of reduced models for robust control and state estimation of a distributed parameter system. Journal of Process Control, 19(3):539-549, 2009.

Z. Emirsjlow and S. Townley. From PDEs with boundary control to the abstract state equation with an unbounded input operator: A tutorial. European Journal of Control, 6(1):27-49, 2000.

K.-J. Engel and R. Nagel. A Short Course on Operator Semigroups. Springer, 2006.

S. J. Farlow. Partial Differential Equations for Scientists and Engineers. Dover Publications, New York, USA, 1993.

S. Grammatico, X. Zhang, K. Margellos, P. Goulart, and J. Lygeros. A scenario approach to non-convex control design: preliminary probabilistic guarantees. In 2014 American Control Conference (ACC), June 4-6, 2014, Portland, Oregon, USA, 2014.

B. V. Keulen. $H_{\infty}$-Control of Distributed Parameter Systems: A state-Space Approach. Springer, New York, USA, 1993.

H. X. Li and C. Qi. Modeling of distributed parameter systems for applications-A synthesized review from time-space separation. Journal of Process Control, 20(8):891-901, 2010.

A. Maidi and J. P. Corriou. Boundary control of nonlinear distributed parameter systems by input-output linearization. In 18th IFAC World Congress, pages 10910-10915, Milan, Italy, 2011. 
R. Padhi and Sk. Faruque Ali. An account of chronological developments in control of distributed parameter systems. Annual Reviews in Control, 33:59-68, 2009.

I. R. Petersen and R. Tempo. Robust control of uncertain systems: Classical results and recent developments. Automatica, 50(5):1315-1335, 2014.

N. N. Puri and G. N. Weygandt. A contribution to the transform calculus used in the synthesis of control systems. Journal of the Franklin Institute, 277(4):337-348, 1964.

W. H. Ray. Advanced Process Control. Butterworths, Boston, 1989.

R. N. P. Singh. A contribution to the theory of non-linear distributed parameter systems. International Journal of Systems Science, 8(1):87-96, 1977.

J. Taler and P. Duda. Solving Direct and Inverse Heat Conduction Problems. Springer, Berlin, 2006.

R. Tempo, G. Calafiore, and F. Dabbene. Randomized Algorithms for Analysis and Control of Uncertain Systems With Applications. Springer, second edition, 2013.

R. Toscano. Structured Controllers for Uncertain Systems. A Stochastic Optimization Approach. Springer, London, 2013.

A. Van de Wouwer, P. Saucez, and W. E. Schiesser. Adaptive method of lines. Chapman and Hall/CRC, 2001. 